\title{
“JOVEN \& FUERTE": PROGRAM FOR YOUNG WOMEN WITH BREAST CANCER IN MEXICO - INITIAL RESULTS
}

\author{
Cynthia Villarreal-Garza ${ }^{1,2,3 *}$, Andrea Castro-SánChez ${ }^{1,4}$, Alejandra Platas $^{1,2}$, Melina Miaja $^{1,3}$,

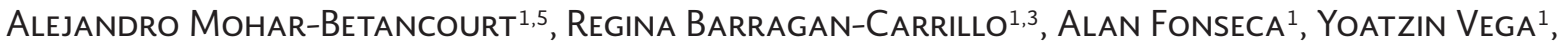 \\ B. Alejandra Martinez-Cannon ${ }^{1,3}$, Dione Aguilar ${ }^{1,3}$, Enrique Bargalló-Rocha ${ }^{1,2}$, \\ Servando Cardona-Huerta ${ }^{3}$, Omar Peña-Curiel ${ }^{1,3}$ AND Juan Matus-Santos ${ }^{1,2}$ \\ ${ }^{1}$ Joven \& Fuerte: Programa para la Atención e Investigación de Mujeres Jóvenes con Cáncer de Mama, Mexico City; \\ ${ }^{2}$ Department of Research and Breast Tumors, Instituto Nacional de Cancerología, Mexico City; ${ }^{3}$ Centro de Cáncer de \\ Mama, Tecnológico de Monterrey, Monterrey, N.L.; ${ }^{4}$ Cátedras CONACYT, Instituto Nacional de Cancerología, Mexico \\ City; ${ }^{5}$ Biomedical Cancer Investigation Unit, Instituto de Investigaciones Biomédicas, Universidad Nacional Autónoma de \\ México, Mexico City, México
}

\begin{abstract}
Despite the high rates of breast cancer among young Mexican women, their special needs and concerns have not been systematically addressed. To fulfill these unsatisfied demands, we have developed "Joven \& Fuerte: Program for Young Women with Breast Cancer in Mexico," the first program dedicated to the care of young breast cancer patients in Latin America, which is taking place at the National Cancer Institute of Mexico and the two medical facilities of the Instituto Tecnológico y de Estudios Superiores de Monterrey. The program was created to optimize the complex clinical and psychosocial care of these patients, enhance education regarding their special needs, and promote targeted research, as well as to replicate this program model in other healthcare centers across Mexico and Latin America. From November 2013 to February 2017, the implementation of the "Joven \& Fuerte" program has delivered specialized care to 265 patients, through the systematic identification of their particular needs and the provision of fertility, genetic, and psychological supportive services. Patients and families have engaged in pedagogic activities and workshops and have created a motivated and empowered community. The program developed and adapted the first educational resources in Spanish dedicated for young Mexican patients, as well as material for healthcare providers. As for research, a prospective cohort of young breast cancer patients was established to characterize clinicopathological features and psychosocial effects at baseline and during follow-up, as a guide for the development of specific cultural interventions addressing this vulnerable group. Eventually, it is intended that the program's organization and structure can reach national and international interactions and serve as a platform for other countries.
\end{abstract}

Key words: Breast cancer. Young women. Survivorship. Program. Mexico. Latin America.

\section{INTRODUCTION}

Young women with breast cancer (YWBC) face particularly challenging age-related health issues and are recognized to be more vulnerable to emotional distress and psychosocial problems, requiring special supportive interventions ${ }^{1,2}$. To optimize the complex

\section{Corresponding author:}

*Cynthia Villarreal-Garza

Batallón San Patricio, 112

Col. Real de San Agustín

C.P. 66278 , San Pedro Garza García, N.L., México

E-mail: cynthia.villarreal@tecsalud.mx clinical care and address the needs of this particular population of women, comprehensive programs for young breast cancer patients have been actively conducted, predominantly in developed countries, such as the United States and Canada ${ }^{1-3}$.

Unfortunately, in low- and middle-income countries, initiatives that have been undertaken to address the particular issues of YWBC are limited because of scarce funding, deficient training among providers, and lack

Received for publication: 03-05-2017

Approved for publication: 06-06-2017

doi: $10.24875 /$ RIC.17002280 
of awareness of the precise needs of young patients which may vary depending on different sociocultural backgrounds. Thus, in limited-resource settings, supportive care for YWBC is often neglected, making it difficult for survivors to rebuild their physical, social, professional, and personal well-being.

In Mexico, where up to $15 \%$ of the total number of newly-diagnosed cases is detected at or before 40 years of age ${ }^{4,5}$, breast cancer among young women represents a significant public health burden. Moreover, in this middle-income country with important socioeconomic limitations, cancer control efforts are predominantly directed to improve medical care, while survivorship issues have not been a priority. Thus, the needs of young Mexican breast cancer patients are often not systematically addressed.

To fulfill these unsatisfied demands, we created "Joven \& Fuerte (Young and Strong): Program for YWBC in Mexico," the first program dedicated to the care of YWBC in Latin America. In this article, we aim to describe the Program's model and to report its initial results.

\section{Program setting}

In November 2013, the "Joven \& Fuerte: Program for YWBC in Mexico" was established at the National Cancer Institute (Instituto Nacional de Cancerología, INCan) in Mexico City. This initiative expanded in August 2014 to two academic clinical sites in the city of Monterrey, Hospital San José and Hospital Zambrano Hellion from the Tecnológico de Monterrey.

This project has been conducted through a joint effort with three key international collaborators: (1) The Program for YWBC at the Dana-Farber Cancer Institute, which has served as a reference and guidance for the start-up and follow-up of the "Joven \& Fuerte" program; (2) the Global Cancer Institute, which has assisted in the development and maintenance of an electronic database for the registry of clinical characteristics and outcome data for research purposes; (3) the Division of Clinical Cancer Genetics at City of Hope Hospital, which has served as a major partner to strengthen the program's genetic cancer risk assessment component and to expand the knowledge of genetic mutations and outcomes in Mexican YWBC.

\section{BASIC PRINCIPLES AND STRUCTURE OF THE PROGRAM}

"Joven \& Fuerte" was developed by a team of healthcare professionals and young breast cancer survivors with the following goals: (1) To optimize the complex clinical and psychosocial needs of young women and their families; (2) to enhance education among patients and healthcare professionals regarding the special needs, along with raising awareness among the general public about timely detection of breast cancer in young women; (3) to promote targeted research addressing unique issues of young Mexican women; (4) to replicate the model's program in other healthcare centers across Mexico and Latin America to reach more vulnerable young patients (Fig. 1).

As for the enrollment of patients into the "Joven \& Fuerte" program, all women aged 40 years or younger are identified in their first clinic visit and/or during multidisciplinary sessions and are immediately referred to an appointment with our program navigator. The navigator meets with the patient, introduces the program, discusses the issues that might be important to YWBC and identifies the patient's special needs (Fig. 2). This facilitator subsequently assists the patient, making referrals to fertility specialists, genetic counseling and emotional supportive services, as appropriate. In addition, the navigator supplies printed teaching material and access to online resources, which were specifically created and adapted for young Mexican patients (see educational component section below). The patients and family members are also invited to participate in educational activities and workshops covering particular topics relevant to young patients and are given a schedule of upcoming events.

To deliver specialized care, such as fertility assessment and genetic counseling, several collaborations have been established to strengthen and accomplish the objectives of the program, by giving YWBC access to resources that otherwise would be prohibitive. For example, the promotion of local alliances between oncology and assisted reproduction centers has increased referrals, as well as the possibility to offer fertility preservation strategies, by partially or totally covering the procedures' costs. As for implementing genetic cancer risk assessment, we have partnered with national and international collaborative institutions to offer genetic testing as part of research 
Figure 1. Chart describing the main goals of the "Joven \& Fuerte" program: Optimization of young women with breast cancer care, education of patients, health care professionals and general public, replication of the model's program and promotion of focused research.

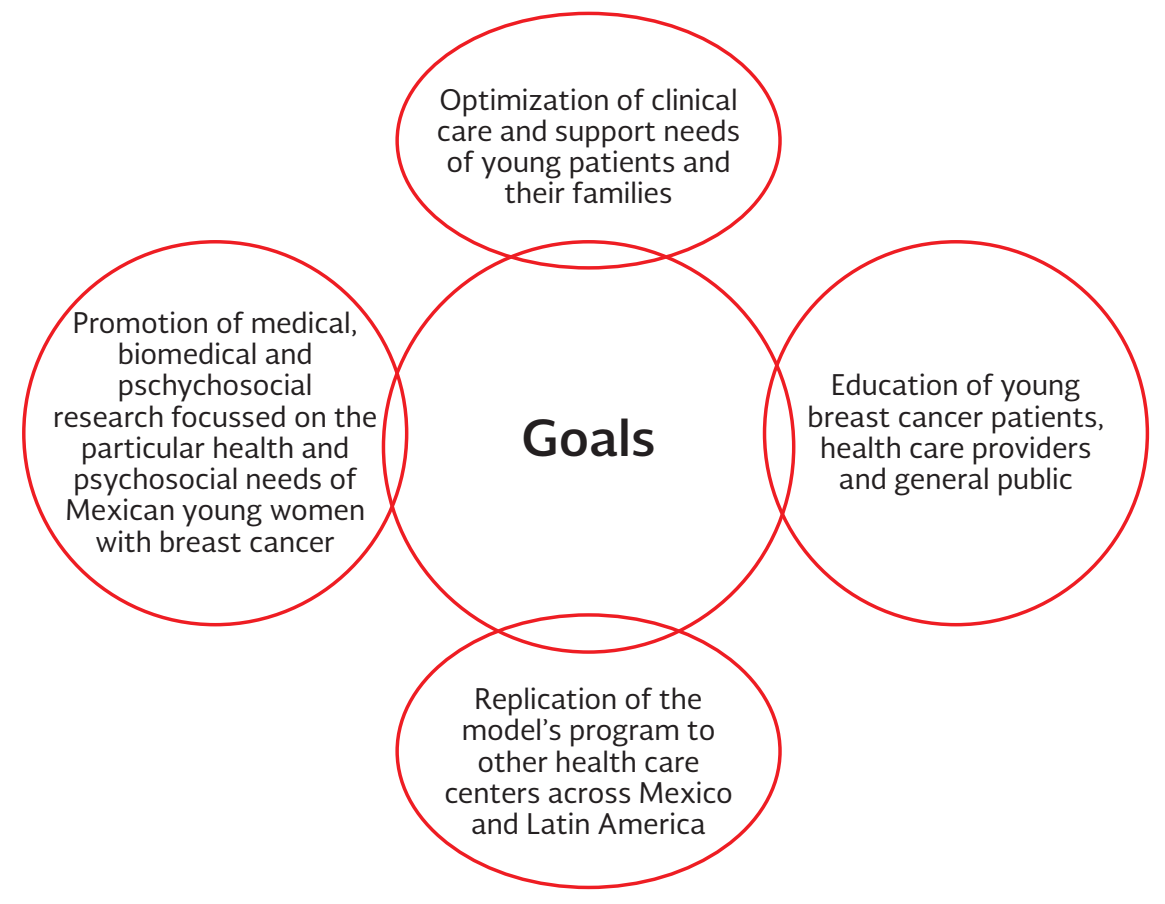

Figure 2. Flowchart of the "Joven \& Fuerte" program integral approach, consisting of five main activities: (1) Patient identification, (2) needs' assessment and timely referral to supportive care services, (3) provision of educational material, (4) invitation to workshops and forums, and (5) invitation to participate in research cohort study.

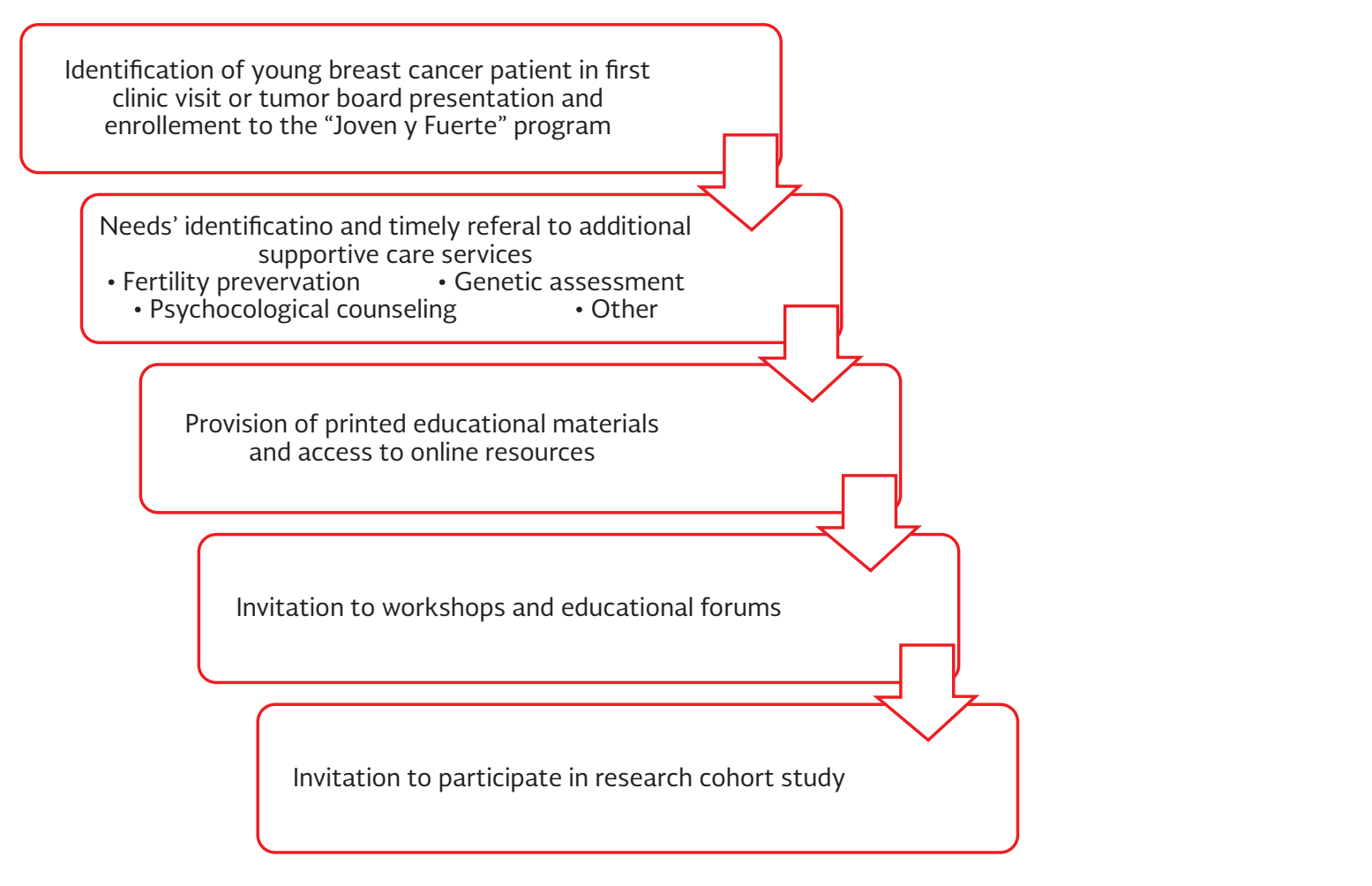


initiatives, which has allowed to overcome barriers associated with the highly expensive and inaccessible costs of genetic testing ${ }^{6}$. In addition, collaborations with nongovernmental organizations are being established to partially or totally cover reconstruction surgery, since the public healthcare system does not include such procedures as a component of breast cancer treatment.

\section{Progress to date}

Up to February 2017, 265 patients had been enrolled in the "Joven \& Fuerte" program: 18 (7\%) patients were timely referred for fertility preservation (with partially or totally covered costs), and $236(89 \%)$ received genetic cancer risk assessment and have been tested for genetic mutations. All patients completed psychological evaluations and 110 (42\%) have received psychological support. 12 seminars and 16 workshops related to specific topics of interest for young women and their families have taken place. Furthermore, eight focus groups were created for the assessment of young Mexican patients' needs to develop educational materials specifically targeted to address those identified concerns.

\section{Educational component}

The "Joven \& Fuerte" program developed and adapted the first resources written in Spanish exclusively dedicated to Mexican young patients by generating an educational manual (Supplementary Figure 1), fact sheets, infographics, website, and mobile application (Supplementary Figure 2). Furthermore, we launched educational online material dedicated to healthcare providers featuring subtitled recorded sessions from national and international experts, checklists, fact sheets, slide presentations and access to key manuscripts and book chapters. The online materials can be accessed through the web address www.jovenyfuerte. com.mx.

\section{Research within the program}

A prospective cohort of newly diagnosed YWBC was established at the three "Joven \& Fuerte" sites. Eligible women answer electronic surveys on relevant topics, such as sociodemographic characteristics, fertility, genetics, and psychosocial needs, among others, while clinicians complete prespecified surveys registering
Table 1. Clinical characteristics of the research cohort of "Joven \& Fuerte"

\begin{tabular}{lc}
\hline Characteristics $(\mathbf{n = 2 4 3 )}$ & $\mathbf{n}(\%)$ \\
\hline Clinical stage at diagnosis & \\
Stage 0 (in situ) & $3(1.2)$ \\
Stage I & $27(11.1)$ \\
Stage II & $98(40.3)$ \\
Stage III & $92(37.9)$ \\
Stage IV & $17(7.0)$ \\
Not classifiable/not available & $6(2.5)$ \\
Biological subtype & \\
HR+/Her2- (luminal) & $126(51.9)$ \\
HR+/Her2+ (luminal Her2+) & $38(15.6)$ \\
HR-/Her2+ (Her2 enriched) & $18(7.4)$ \\
HR-/Her2- (triple negative) & $56(23.0)$ \\
Not available/unknown & $5(2.1)$ \\
\hline
\end{tabular}

clinicopathologic features and outcomes. Patients are evaluated at diagnosis, after 6 months, and annually for 5 years. Substudies assessing changes in cognition, sexual function and satisfaction, quality of life, and depression/anxiety are being carried out, and biologic samples are obtained and stored frozen for future research.

Until February 2017, 243 patients have been included in the cohort, with a median age at diagnosis of 36 years (range $21-40$ years). Table 1 enlists the clinical stage at breast cancer diagnosis and the biological subtype. To date, nine patients (4\%) have developed distant recurrences, and 12 patients (5\%) have died as a consequence of breast cancer, with a median follow-up of 17 months. The remaining research variables are still under collection and analysis.

As for research productivity, eight peer-reviewed manuscripts have been published and seven international abstracts presented. During this period, "Joven \& Fuerte" has been awarded with four grants: CONACYT (National Council for Science and Technology), Mexico; Fundación CIMAB, Mexico; Susan G. Komen for the Cure, USA; and Avon, Mexico.

Several research lines are being developed by clinical investigators affiliated to the program with particular interest in YWBC. Supplementary Table 1 contains in detail the research topics and their corresponding investigators. It is envisaged that research on these topics related to breast cancer will enrich our knowledge on the particular demands of YWBC in Mexico and will help develop culturally specific interventions to improve overall psychosocial and medical outcomes of this vulnerable group. 


\section{Community}

"Welcome, you have reached your shelter" is the catchphrase of the "Joven \& Fuerte" program that encourages YWBC to become fully involved in the program's activities. At present, two large young breast cancer communities in Mexico City and Monterrey, guided and supported by healthcare professionals of the program, are proudly motivated and empowered not only to face their own challenges from a more assertive and forceful standpoint, but also to encourage newly diagnosed breast cancer patients to become part of this strong and hopeful entity.

\section{CHALLENGES AND FUTURE DIRECTIONS}

Funding is a constant struggle for continuity of our program, which currently mostly relies on limited grants for the financial support of its activities, such as governmental contributions and donations from nongovernmental organizations. The latter conveys two major potential problems: First, lack of sustainability and risk of discontinuity, and second, vulnerability to patient saturation because of restricted human and material resources. Interinstitutional and international collaborations are taking place to make the most of available resources, prevent repeating efforts, and promote fund sharing. Furthermore, there is a need to ensure the continuity and expansion of research within the program through collaborations with national and international research groups who are deeply motivated and interested in studying breast cancer in young women and their needs.

It is intended that the program's organization and structure reach national and international interactions and serve as a model for other Latin American countries. The Avon-Mexico grant is designated to guarantee that the program is reproduced in five additional centers across Mexico.

In conclusion, the "Joven \& Fuerte" program has delivered, for the first time in Mexico, standardized and specialized care for YWBC, through the systematic identification of their particular needs and the provision of fertility, genetic, and emotional supportive services. The program has also provided patients, and their families with educational resources in Spanish specifically adapted for their easy comprehension and use. From the healthcare provider's standpoint, the program has contributed to continuous medical education through the design and production of specialized teaching material focused on young women's particular needs, with the ultimate goal of improving patient-centered care.

In addition, the prospective collection of medical and psychosocial data will guide culturally directed interventions to enhance breast cancer survivorship care for the Latin American region. Remarkably, this motivated and empowered community of patients and family members, partnered with their healthcare team, have found hope on the face of cancer. We will continue to direct our efforts to accomplish the "Joven \& Fuerte" program goals, targeted to reach more young patients and their beloved ones.

\section{ACKNOWLEDGMENTS}

The authors would like to thank all the members of our team who have participated in the creation, implementation and sustainability of the Joven \& Fuerte program: Abelardo Meneses-Garcia, Abish Romero, Ana Burgos-Gonzalez, Ana Platas, Alexandra Bukowski, Ann H. Patridge, Bertha Aguilar, Brizio Moreno, Carmen Lizette Galvez-Hernandez, Carlos Navarro, Christian Aguila, Cristina Aguilar-González, Cristina MagallanesHoyos, César Marquez-Perez, Ellen Warner, Jaime Hidalgo-Carrera, Janeth Castro-Carrasco, Jeffrey N. Weitzel, Jessica St Louis, Judy-Ann Chapman, Karla Unger-Saldaña, Karina Tellez, Kathleen R. Blazer, Laura Ortega-Leonnard, Lenny Gallardo, Liliana GómezFlores-Ramos, Mauricio Canavati, Paul E. Goss, Paula Cabrera-Galeana, Pier Ramos-Elias, Raquel Menendez, Robin Shaw, Rosa María Alvarez, Sandra Patricia Rivera-Sandoval, Sandra Gonzalez, Talia Wegman, and the Global Cancer Institute, the Breast Cancer and Genetics Departments of the Instituto Nacional de Cancerología, Mexico City, and the Tecnológico de Monterrey, Monterrey, NL, Mexico.

\section{SUPPLEMENTARY DATA}

Supplementary data is available at Revista de Investigación Clínica online (www.clinicalandtranslationalinvestigation.com). These data are provided by the corresponding author and published online for the 
benefit of the reader. The contents of supplementary data are the sole responsibility of the authors.

\section{REFERENCES}

1. Villarreal-Garza C, Martinez-Cannon BA, Platas A, Ramos-Elias P. Specialized programs to support young women with breast cancer. Curr Opin Support Palliat Care. 2015;9:308-16.

2. Partridge AH, Ruddy KJ, Kennedy J, Winer EP. Model program to improve care for a unique cancer population: Young women with breast cancer. J Oncol Pract. 2012;8:e105-10.

3. Ali A, Warner E. Pynk: Breast cancer program for young women. Curr Oncol. 2013;20:e34-9.
4. Villarreal-Garza C, Aguila C, Magallanes-Hoyos MC, Mohar A, Bargalló $\mathrm{E}$, Meneses A, et al. Breast cancer in young women in Latin America: An unmet, growing burden. Oncologist. 2013;18 Suppl:26-34.

5. Villarreal-Garza C, Mohar A, Bargallo-Rocha JE, Lasa-Gonsebatt F, Reynoso-Noverón N, Matus-Santos J, et al. Molecular subtypes and prognosis in young Mexican women with breast cancer. Clin Breast Cancer. 2016;17:e95-102.

6. Villarreal-Garza C. Steps to address the profound disparity in access to genetic cancer risk assessment in Mexico: Documenting Hereditary Breast and Ovarian Cancer and the Beginning of a Prevention Program. Presented at the $7^{\text {th }}$ AACR Conference on The Science of Health Disparities in Racial/Ethnic Minorities and the Medically Underserved, November 9-12, San Antonio, TX, Abstract IA23; 2015. 